\title{
Reacciones Adversas Medicamentosas en Pacientes que consultaron a Instituciones Prestadoras de Servicios en Pereira, Colombia
}

\author{
Jorge E. Machado-Alba ${ }^{1}$ y Juan C. Moncada-Escobar ${ }^{2}$ \\ 1 Médico, Especialista en Epidemiología. Profesor Facultad de Ciencias de la Salud, Universidad \\ Tecnológica de Pereira. Departamento de Farmacoepidemiología Audifarma S.A. \\ E-mail: jormach@telesat.com.co \\ 2 Médico, Especialista en Auditoria en Salud y Gerencia de la Calidad. Departamento de \\ Farmacoepidemiología Audifarma S.A. E-mail: juancarlos@audifarma.com.co
}

Recibido 20 Enero 2006/Enviado para Modificación 3 Junio 2006/Aprobado 20 Junio 2006

\section{RESUMEN}

Objetivo Describir las reacciones adversas medicamentosas (RAM) y analizar factores asociados con su aparición en pacientes consultantes en servicios de urgencias de dos clínicas.

Métodos Estudio de farmacovigilancia, descriptivo, prospectivo, de personas que consultan en servicios de urgencias de Clínica los Rosales y Unidad Atención Básica Salud Total en Pereira, entre julio y septiembre de 2005, por cualquier síntoma relacionado con toma de medicamentos.

Resultados Total 91 notificaciones. El 62,6 \% en mujeres, edad promedio 36,3 $\pm 22,4$ años, rango: 0-85 años. Grupos farmacológicos más representativos: antibióticos $(24,2 \%)$, AINES (17,6 \%), analgésicos $(9,9 \%)$ y antidiabéticos (8,8 \%). El 39,6\% de pacientes utilizaban solo un medicamento, promedio de fármacos por paciente fue $2,4 \pm 1,5$. El 25,3\% se automedicó. Las RAM más frecuentes fueron urticaria alérgica (31,9\%), hipoglicemia (8,8 \%), gastritis agudas (6,6 \%), edema angioneurótico (5,5\%) y anafilaxia $(4,4 \%)$. El $13,2 \%$ de las RAM fueron graves y el $54,9 \%$ fueron predecibles. Se clasificaron como definitivas el $14,3 \%$ y probables el $75,8 \%$. El 14,3\% de los pacientes tenían antecedentes de RAM. El costo promedio de atención fue US \$78,1. El uso de analgésicos estuvo relacionado con anafilaxia; de antibióticos con urticaria alérgica; de AINES con gastritis y hemorragia digestiva; de antidiabéticos con hipoglicemia; de psicofármacos con síntomas neurológicos, y de warfarina con hemorragias.

Conclusiones Se determinaron los factores asociados de manera más importante con RAM en pacientes que acudieron a servicios de urgencias. Se debe prestar especial atención al uso de AINES, warfarina, metoclopramida, dipirona, psicofármacos, hipoglicemiantes y antiinfecciosos, a la historia previa de RAM, la edad mayor de 55 años y presentar síntomas en piel, digestivos y neurológicos. 
Palabras Clave: Reacción, medicamentosa, costos, atención, salud (fuente: DeCS, BIREME)

\begin{abstract}
Adverse drug reactions in patients attending in emergency service Objective Describing adverse drug reactions (ADR) and analyzing the factors associated with their appearance in patients attending two hospitals' emergency services.

Methods Descriptive, medicine-surveillance study of people consulting the emergency service at the Rosales hospital and the basic total health attention unit in Pereira, Colombia, between July and September 2005 for any symptom related to taking medicine. Naranjo's Adverse Drug Reaction probability scale was used.

Results A total of 91 patients were found; $62,6 \%$ were women having an average age of $36,3 \pm 22,4$ (range $0-85$ ). Antibiotics $(24,2 \%)$ NSAIDs $(17,6$ $\%)$, analgesics $(9,9 \%)$ and antidiabetic agents $(8,8 \%)$ were the most used drugs. $39,6 \%$ of the patients only used one medicine; the average of drugs per patient was $2,4 \pm 1,5$. Self-medication was present in $25,3 \%$. The most common ADRs arose from urticaria (31,9\%), hypoglycaemia $(8,8 \%)$, gastritis (6,6 \%), angioedema (5,5\%) and anaphylaxis (4,4\%). 13,2 \% of ADRs were serious and $54,9 \%$ were avoidable. $14,3 \%$ of ADRs were classified as being definite and $75,8 \%$ probable. Only $14,3 \%$ of patients had a past history of ADR. The average cost of being attended was US\$ 78,1 dollars. Analgesic use was associated with anaphylaxis, antibiotics with urticaria whilst NSAIDs was associated with gastritis and gastrointestinal bleeding, antidiabetic agents with hypoglycaemia, antipsychotic and antidepressants agents with neurological symptoms and warfarin with bleeding.

Conclusions The most important ADR-associated risk factors in patients attending emergency services were determined. Special care must be paid to patients using NSAIDs, warfarin, metoclopramide, metamizole, antipsychotic and antidepressants agents, antidiabetic agents and antibiotics, having a past history of ADR, being more than 55 years old and presenting urticaria, digestive and/or neurological symptoms
\end{abstract}

Key Words: Adverse drug reaction, health care costs (source: DeCS, NLM).

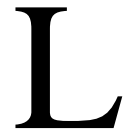
as reacciones adversas medicamentosas (RAM) son eventos que pueden afectar gravemente la salud de las personas que consumen fármacos con fines terapéuticos, diagnósticos o profilácticos. Diversos estudios en otros países han puesto de manifiesto que entre un 1,7 y un $28 \%$ de las atenciones en hospitales pueden deberse a efectos indeseables. Las tasas de mortalidad por RAM pueden ir desde 0,12 hasta 0,32 \% (1-8). Se calcula que entre el 59 y $81 \%$ de las reacciones adversas a medicamentos son 
prevenibles o completamente evitables (3-5,9-16). Entre el 0,34 \% y el $23 \%$ de las RAM pueden terminar en hospitalización $(9,13)$.

Las complicaciones gastrointestinales son las causas más comunes de admisión por RAM, incluyendo el sangrado digestivo, seguidas de alteraciones en piel, sistema nervioso y aparato cardiovascular (3,16-19). Los medicamentos más frecuentemente asociados con ingresos por RAM son los AINES, seguidos de anticoagulantes, digitálicos, antiarrítmicos, antineoplásicos, antitrombóticos, antibióticos y antihipertensivos, incluyendo los diuréti$\cos (3,4,12,16-19)$.

Se ha encontrado que hasta el $68,7 \%$ de las RAM son tipo A (aumentadas) y el 31,3 \% del tipo B (bizarras) según la clasificación de Rawlins y Thompson. Factores que con frecuencia se asocian a RAM son género femenino, edad, tratamiento con citostáticos o antiinfecciosos, antecedentes de RAM y polimedicación $(2,3,17,20)$.

En Colombia no hay publicados informes de este tipo por lo que el presente estudio pretende describir las RAM y analizar los factores que se asocian con su aparición en pacientes que consultan a los servicios de urgencias de las clínicas Los Rosales y Unidad Atención Básica Salud Total en Pereira, entre julio y septiembre de 2005. Además busca determinar la frecuencia de uso de formatos de notificación de efectos indeseables entre los médicos de estas clínicas y hacer un análisis de los costos de atención en salud de estos pacientes.

\section{MATERIALES Y MÉTODOS}

Se trata de un estudio descriptivo y prospectivo de farmacovigilancia. Se consideraron sospechosas todas las personas de cualquier edad, de ambos sexos, que consultaron por cualquier problema médico relacionado con la toma de medicamentos en los servicios de urgencias de las clínicas Los Rosales y Unidad Atención Básica Salud Total en Pereira, entre julio y septiembre de 2005.

Para facilitar la búsqueda, se entregó a los médicos un listado de probables síndromes considerados alerta para RAM. Cada paciente sospechoso fue sometido a un protocolo de preguntas consignado en un formato para reporte de RAM, que recogía datos sociodemográficos, costo de la atención, y las variables relacionadas con el medicamento, dosis y la RAM. Cuando hubo necesidad uno de los investigadores entrevistó de nuevo a los pacientes. 
A pesar de que existen otras clasificaciones para RAM, se tomó la más simplificada utilizada por Rawlins y Thompson que las ordena en A y B (2) y para los medicamentos se utilizó la clasificación ATC (Anatómica, Terapéutica y Química). La relación de causalidad entre efectos indeseables y el medicamento se estableció mediante la metodología para estimación de probabilidad de reacción adversa, diseñada por Naranjo (21). Se clasificó el desenlace en "leve" para los pacientes dados de alta después de la atención, "moderado" para los que estuvieron varias horas en observación y "grave” para aquellos que requirieron hospitalización.

El estudio fue revisado y aprobado por el Comité de Bioética de la Universidad Tecnológica de Pereira. A todos los pacientes se les informó y se obtuvo un consentimiento informado (22). Los datos se almacenaron, procesaron y analizaron con el paquete estadístico SPSS 13.0 para Windows. Se utilizó la prueba ji al cuadrado para la comparación de variables. Se hizo un análisis multivariado utilizando regresión logística binaria. Se determinó un nivel de significancia estadística en $\mathrm{p}<0,05$.

\section{RESULTADOS}

Se diligenciaron y analizaron 102 formatos de los cuales 11 se descartaron por contener síntomas propios de una enfermedad de base del paciente y los otros 91 contenían información compatible con una reacción adversa medicamentosa. La prevalencia de consulta por RAM en servicios de urgencias fue de $0,46 \%$.

El 62,6 \% de los casos fueron mujeres, la media de edad fue de 36,3 \pm 22,4 años, con un rango de 0-85 años. Los grupos de medicamentos más representativos según clasificación ATC fueron los antiinfecciosos de uso sistémico (24,2 \%), los antiinflamatorios y antirreumáticos no esteroides $(17,6$ $\%)$, los analgésicos (9,9\%), los medicamentos usados en diabetes (8,8 \%), antieméticos y antinauseosos (4,4 \%), agentes antitrombóticos (4,4\%), antihipertensivos $(4,4 \%)$, antipsicóticos $(4,4 \%)$ y antidepresivos $(4,4)$.

El 74,7 \% de los pacientes recibieron el medicamento por vía oral y el 20,9 \% por vía parenteral. Las indicaciones más frecuentes para la prescripción de medicamentos fueron analgesia y antipiresis con 26,4 \%, infección bacteriana sistémica con 25,3 \%, diabetes mellitus con 8,8 \%, hipertensión arterial con 6,6 \%, trastornos trombo-embólicos con 4,4 \% y trastorno gástrico funcional con $4,4 \%$. 
El 39,6 \% de los pacientes utilizaban solamente un medicamento; la media de fármacos por paciente fue de 2,4 $\pm 1,5$. El 25,3 \% de los pacientes se automedicó. Las reacciones adversas más frecuentes según el sistema comprometido pueden verse en la Tabla 1 . El 13,2 \% fueron reacciones adversas graves que requirieron hospitalización, el $22 \%$ moderadas y el 64,8 \% leves; no se presentó ninguna muerte secundaria a la utilización de medicamentos.

Un 54,9 \% de las RAM fueron del tipo A y el restante 45,1 \% del tipo B. El 75,8 \% de RAM fueron clasificadas como probables, el 14,3 \% definitivas, el 6,6 \% posibles y 3,3 \% fueron dudosas. El 14,3\% de los pacientes tenían antecedentes de haber presentado reacciones adversas similares al mismo medicamento. Los costos de atención variaron según la gravedad de la reacción adversa atendida. Se tuvo en cuenta la tasa representativa del mercado en diciembre 2005 de 2276 pesos por dólar (Tabla 2).

Tabla 1. Reacciones adversas medicamentosas más frecuentes según sistema comprometido

\begin{tabular}{lcc}
\hline \multicolumn{1}{|c}{ Reacción Adversa Medicamentosa } & Frecuencia & $\%$ \\
\hline Cardiovascular & & \\
\hline Hipertensión secundaria & 2 & 2,2 \\
\hline Neurológica & 3 & 3,3 \\
\hline Cefalea Inducida por drogas & 3 & 3,3 \\
\hline Distonía inducida por drogas & 3 & 3,3 \\
\hline Parkinsonismo secundario inducido & 3 & 3,3 \\
\hline Somnolencia & 3 & 3,3 \\
\hline Otros vértigos periféricos & & \\
\hline Gastrointestinal & 6 & 6,6 \\
\hline Otras gastritis agudas & 3 & 3,3 \\
\hline Nausea y vomito & 2 & 2,2 \\
\hline Gastritis aguda hemorrágica & & \\
\hline Dermatológicas & 29 & 31,9 \\
\hline Urticaria alérgica & 5 & 5,5 \\
\hline Edema angioneurótico & & \\
\hline Hematológicas & 2 & 2,2 \\
\hline Hematuria, no especificada & 2 & 2,2 \\
\hline Hemorragia, no clasificada & & \\
\hline Endocrinológicas & & \\
\hline Hipoglucemia sin coma, inducida por & & \\
\hline Otros & & \\
\hline Choque anafiláctico & & \\
\hline & & \\
\hline
\end{tabular}


Tabla 2. Costos de la atención según gravedad de la RAM

\begin{tabular}{lcc}
\hline \multicolumn{1}{c}{ Desenlace } & $\begin{array}{c}\text { Número } \\
\text { pacientes }\end{array}$ & $\begin{array}{c}\text { Costo promedio } \\
\text { US dólar }\end{array}$ \\
\hline Alta & 59 & 32,2 \\
Observación & 20 & 105 \\
Hospitalización & 12 & 259 \\
Total & 91 & 78,1 \\
\hline
\end{tabular}

No se encontró ninguna asociación estadísticamente significativa entre el sexo y las demás variables. La edad más avanzada se asoció con mayor número de medicamentos por paciente $(\mathrm{p}<0,03)$, con la RAM presentada $(p<0,003)$ y con el costo de la atención $(p<0,03)$. No hubo asociaciones significativas con la automedicación $(\mathrm{p}>0,9)$. La anafilaxia se relacionó con hospitalización. Solo la hipoglicemia se asoció con antecedentes de RAM $(\mathrm{p}<0,01)$.

En el análisis multivariado se encontró que las RAM en sistema nervioso se asociaron con edad mayor de 55 años $(\mathrm{p}<0,02)$, con uso de antieméticos $(\mathrm{p}<0,001)$ y psicofármacos $(\mathrm{p}<0,000)$; los antibióticos se asociaron con urticaria alérgica $(\mathrm{p}<0,04)$, los AINES con trastornos digestivos $(\mathrm{p}<0,006)$ y urticaria alérgica $(\mathrm{p}<0,000)$; la warfarina con hemorragias y hospitalización $(\mathrm{p}<0,03)$.

De los 31 médicos encuestados, solo el 16,1 \% conocían un formato de reporte de RAM, e igual número habían reportado alguna vez.

\section{DISCUSIÓN}

Se consiguió describir las reacciones adversas más comunes en los pacientes que consultaron a los dos servicios de urgencias. El número de casos es suficiente para establecer las asociaciones más representativas. Los hallazgos son útiles para poblaciones de características similares que acudan a servicios de urgencias y no son aplicables a pacientes hospitalizados.

Los resultados encontrados son acordes con los de la literatura científica sobre el tema $(4,17,23)$, la prevalencia de consulta por reacciones adversas a servicios de urgencias está en el rango bajo $(7,24)$, posiblemente porque la mayoría de estas son leves (64,8 a 70,8 \%), por lo cual consultan a servicios ambulatorios y no de urgencias $(16,25)$.

La edad promedio es menor que la reportada por la literatura, seguramente porque la población colombiana es más joven que aquella de la lite- 
ratura europea. La edad de los pacientes con RAM tipo A fue significativamente mayor que la de aquellos con RAM tipo $B(p<0,000)(3,26)$. Fue evidente que a mayor edad, tomaban más medicamentos y se elevaba su riesgo de sufrir RAM, relacionado con la polifarmacia del adulto mayor (20).

A diferencia de otros reportes, la automedicación no ofreció ningún riesgo adicional de tener una RAM $(\mathrm{p}>0,6)$. En pacientes con diabetes mellitus se encontró asociación entre la RAM y la polifarmacia que potencia la hipoglicemia motivo de consulta, ya descrita por otros $(\mathrm{p}<0,009)(25)$.

No se encontró relación alguna entre las reacciones adversas y el sexo de los pacientes, a diferencia de los estudios de De Abajo y Medina que señalan el género femenino como un factor de riesgo importante $(16,17)$, pero no hubo ningún hallazgo adicional que nos de una explicación a esto. Se consideró que $13,2 \%$ es un elevado porcentaje de hospitalización, estando por arriba de reportes españoles y franceses $(1,1 \%, 9,6 \%$ y 6,1 \% respectivamente) $(2,6,30)$ y por debajo del $24 \%$ registrado en Canadá (11).

El costo de la atención fue más alto en pacientes de mayor edad $(\mathrm{p}<0,03)$, en los que presentaron anafilaxia o en aquellos con hemorragias $(p<0,00)$, en quienes tuvieron RAM graves $(p<0,000)$ y en RAM definitivas $(p<0,000)$. Los costos son menores que los reportados en otros países (25-27).

La vía de administración predominante fue la oral, característica en pacientes ambulatorios. Se corroboró que el uso de analgésicos estuvo relacionado con anafilaxia; de AINES y antibióticos con urticaria alérgica; de antidiabéticos con hipoglicemia; de psicofármacos con síntomas neurológicos, y de warfarina con hemorragias $(\mathrm{p}<0,00)$ como ya ha sido descrito (9).

En este estudio se establece la asociación entre AINES por vía oral con gastritis y hemorragia digestiva $(\mathrm{p}<0,006)$, como aparece en la mayoría de literatura consultada $(4,8,28,31)$. La asociación entre antiinfecciosos por vía parenteral con RAM tipo B $(\mathrm{p}<0,02)$, puede evidenciar el mayor riesgo que implica utilizar antibióticos parenterales en pacientes ambulatorios.

Se identificaron los factores más importantes asociados con RAM en pacientes que acudieron a servicios de urgencias. Se debe prestar especial atención al uso de AINES, warfarina, metoclopramida, dipirona, psicofármacos, hipoglicemiantes y antiinfecciosos, a la historia previa de RAM, la edad mayor de 55 años y presentar síntomas en piel, digestivos y neurológicos. 
Debido al pobre desarrollo de la notificación de RAM y de programas de farmacovigilancia en Colombia, éste trabajo puede contribuir a generar un conocimiento sobre las características más comunes de estos eventos y motivar la implementación de políticas institucionales que favorezcan su reporte e investigación (25).

Agradecimientos. A la Universidad Tecnológica de Pereira y Audifarma S.A. A Diana González, Melissa Rosero y Darlin Agualimpia, estudiantes de medicina.

\section{REFERENCIAS}

1. Guemes M, Sanz E, Garcia M. Adverse reactions and other drug-related problems in an emergency service department. Rev Esp Salud Pública. 1999; 73:5118.

2. Laporte J.R, Tognoni G. Principios de epidemiología del medicamento. Barcelona: Salvat Medicina. 2 edición; 1993. pp. 95-107.

3. Pirmohamed M, James S, Meakin S, Green C, Scout A, Walley T, Farrar K, Park $\mathrm{K}$, Breckenridge A. Adverse drug reactions as cause of admission to hospital: prospective analysis of 18.820 patients. BMJ 2004; 329:15-19.

4. Pouyanne P, Haramburu F, Imbs J, Bégaud B. Admissions to hospital caused by adverse drug reactions: cross sectional incidence study. BMJ 2000; 320:1036

5. Olivier P, Boulbes O, Tubery M, Carles P, Montastruc JL, Lapeyre-Mestre M. Preventability of adverse effects in a medical emergency service. Therapy 2001; 56:275-8.

6. Lazarou J, Pomeranz BH, Corey PN. Incidence of adverse drug reactions in hospitalizad pateints - a meta-analysis of prospective studies. JAMA 1998; 279:1200-5.

7. Einarson TR, Drug-related hospital admissions. Ann Pharmacother. 1993; 27:83240.

8. Schneeweiss S, Hasford J, Göttler M, Hoffman A, Riethling AK, Avorn J. Admissions caused by adverse drug events to internal medicine and emergency departments in hospitals: a longitudinal population-based study. Eur J Clin Pharmacol. 2002; 58:285-91.

9. Patel P, Zed PJ. Drug-related visits to emergency department: how big is the problem? Pharmacotherapy. 2002; 22:915-23.

10. Winterstein AG, Sauer BC, Hepler CD, Poole C. Preventable drug-related hospital admissions. Ann Pharmacother. 2002; 36:1238-48.

11. Ruedy J, Ogilvie R. Adverse events: past and future. CMAJ. 2004; 171:10.

12. Forster A, Heather C, Menard A, Dupuis N, Chernish R, Chandok N, Khan A, van Walraven C. Adverse events among medical patients after discharge from hospital. CMAJ. 2004; 170:345-9.

13. Honigman B, Lee J, Rothschild J, Light P, Pulling R, Yu T, Bates D. Using computerized data to identify adverse drug events in outpatients. J Am Med Inform Assoc. 2001; 8:254-66. 
14. Vincent C, Neale G, Woloshynowych M. Adverse events in British hospitals: preliminary retrospective record review. BMJ 2001; 322:517-9.

15. Chan M, Nicklason F, Vial JH. Adverse drug events as a cause of hospital admission in the elderly. Intern Med J. 2001; 31:199-205.

16. De Abajo FJ, Frias J, Lopo CR, Garijo B, Castro MA, Carcas A, Juarez S, Gil A. Las reacciones adversas a medicamentos como motivo de consulta al servicio de urgencias de un hospital general. Med Clin. 1989; 92:530-5.

17. Medina MA, Puche E, Luna J. Factores asociados con la presentación de reacciones adversas a medicamentos en pacientes que acuden al servicio de urgencia de un hospital general: estudio de casos y controles. Aten Primaria. 2000;26:42-4.

18. Anónimo. Nueve años de tarjeta amarilla. Butll Groc. 1992;5:1-4.

19. Anónimo. Quince años de tarjeta amarilla. Butll Groc. 1998;11:1-4.

20. Hafner JW, Belknap SM, Squillante MD, Bucheit KA. Adverse drug events in emergency department patients. Ann Emerg Med. 2002;39:258-67.

21. Naranjo CA, Busto U, Sellers E, Sandor P, Ruiz I, Roberts E, Janecek E, Domecq C, Greenblatt J. A method for estimating the probability of adverse drug reactions. Clin pharmacol Ther. 1981; 188:7-12.

22. Ministerio de Salud de Colombia. Dirección de Desarrollo Científico y Tecnológico. Normas científicas, Técnicas y Administrativas para la Investigación en Salud. Resolución No. 8430 de 1993.

23. Hoffman J, Weir C, Bennett C, Hurdle J. High rates of adverse drug events in a highly computerized hospital. Arch Intern Med. 2005; 165:1111-16

24. Gutierrez S, Repetto M. Episodios Adversos a medicamentos detectados en dos servicios de internación pediátrica del Centro Hospitalario Pereira Rossell. Arch Pediatr Urug. 2004; 75:307-15.

25. Muñoz MJ, Ayani I, Rodríguez-Sasiain JM, Gutiérrez G, Aguirre C. Monitorización en un servicio de urgencias de reacciones adversas causadas por medicamentos en niños y adultos. Med Clin. 1998; 111:92-8.

26. Segura O, Maldonado C. Las reacciones adversas a medicamentos: una aproximación desde el punto de vista económico. Biomédica. 2003; 23:401-7.

27. Dennehy CE, Kishi DT, Louie C. Drug-related illness in emergency department patients. Am J Health Syst Pharm. 1996; 53:1422-6.

28. Chassaignon C, Letoumelin P, Pateron D. Upper gastrointestinal hemorrhage in Emergency Departments in France: causes and management. Eur J Emerg Med. 2003; 10:290-5.

29. Dunn N. Adverse drug event. BMJ 2003; 326:1018.

30. Ibáñez L, Laporte JR, Carné X. Adverse drug reactions leading to hospital admisión. Drug Saf. 1991; 6:450-59.

31. The Centers for Education and Research on Therapeutics (CERTs). Risk assessment of drugs, biologics and therapeutic devices: present and future issues. Pharmacoepidemiology and drug safety. 2003; 12:653-662. 